\title{
Timpanoplastías en adultos en el Complejo Asistencial Dr. Sótero del Río (CASR): Revisión de 5 años
}

\section{Tympanoplasty in adults at the Complejo Asistencial Dr. Sótero del Rio (CASR): Review of 5 years experience}

\author{
Francisco De la Paz P1', Javiera Pardo J², Roberto Fernández G .
}

\begin{abstract}
RESUMEN
Introducción: En la residencia de tercer año de la beca de otorrinolaringología de la Pontificia Universidad Católica de Chile se realiza el "comité de oído" en el Hospital Clínico UC y el Complejo Asistencial Dr. Sótero del Rio (CASR) lo que nos ha permitido una experiencia. Quisimos revisar los resultados de las timpanoplastías realizadas en este último en un plazo de 5 años.

Objetivo: Los objetivos de este estudio son identificar y describir las características epidemiológicas de los pacientes sometidos a timpanoplastía atendidos en el CASR, además evaluar la frecuencia de utilización de diferentes técnicas quirúrgicas y sus resultados anatómicos y auditivos.

Material y método: Se realizó un estudio retrospectivo y descriptivo. Se revisaron 101 fichas de los pacientes mayores de 15 años con diagnóstico de OMC sometidos a timpanoplastía atendidos en el CASR entre enero de 2000 y diciembre de 2004.

Resultados: Éxito anatómico en el $79 \%$ de los casos. En relación al resultado auditivo $62 \%$ de los pacientes mejoró más de $10 \mathrm{~dB}$ su audición y $30 \%$ la mantuvo igual, lo que determina $92 \%$ de los pacientes con buen resultado auditivo.

Conclusiones: Estos resultados corresponden al rendimiento de la timpanoplastía practicada en su mayoría por residentes de $3^{\circ}$ año y otorrinos generales con distintos niveles de formación en otocirugía. Esto podría explicar la leve disminución en el éxito anatómico, comparado con series nacionales.
\end{abstract}

Palabras clave: Timpanoplastía, otitis media crónica, resultados, adultos.

\begin{abstract}
Introduction: In the third year of the residency of otolaryngology in the Pontificia Universidad Católica de Chile the residents forms part of the "ear committee" of the Hospital Clínico UC and the Complejo Asistencial Dr Sotero del Rio (CASR), is that why we want to review the timpanoplasty results with a follow up of 5 years.

Aim: The aim of this study is identify and show the epidemiologic data of the patients who was operated of timpanoplasty in the CASR, also review the different surgical techniques and the anatomic and auditive results.
\end{abstract}

Médico del Servicio de Otorrinolaringología, Hospital Sótero del Río.

2 Médico Cirujano, Pontificia Universidad Católica de Chile. 
Material and method: A retrospective, descriptive study was made. We review 101 records of all patients older than 15 years old, who presented chronic otitis media and underwent tympanoplasty, between January 200 and December 2004.

Results: Anatomical success in $79 \%$ of all cases. $62 \%$ of all cases were better than $10 \mathrm{~dB}$ from base hearing, and 30\% keep the same level of hearing, which determines $92 \%$ of all the patients with a good hearing outcome.

Conclusions: These results correspond to the performance of tympanoplasty practiced mostly by residents of 3rd year and general ENT with different levels of training in otologic surgery. This may explain the slight decrease in the anatomic success compared with national series.

Key words: Tympanoplasty, chronic otitis media, results, adults.

\section{INTRODUCCIÓN}

El concepto de reparación quirúrgica de la membrana timpánica con un injerto de piel se atribuye usualmente a Berthold, quien en agosto del año 1878 reportó la primera timpanoplastía. Sin embargo, existe controversia, ya que un segundo autor, Ely, habría realizado la primera cirugía sin reportar en junio del mismo año.

Posteriormente, en 1952, Wullstein y Zöllner presentaron en el Congreso Alemán de Reichenhall los conceptos quirúrgicos actuales de timpanoplastía. Wullstein, por su parte, utilizó injertos de piel libre, en tanto, Zöllner piel pediculada. En el mismo año Wullstein usó el término "tympanoplastik" para describir las diferentes técnicas de reconstrucción de la membrana timpánica. Al año siguiente definió el objetivo de la timpanoplastía como "...su objetivo es quirúrgico, la mayoría de las veces una cirugía en un solo tiempo, que reconstruya la audición creando una nueva membrana timpánica para cualquier otitis media crónica". El mismo año House escribió: "el pensa- miento de usar un injerto de piel delgada sobre la región del oído medio me parece realizable".

En 1956 Wullstein clasifica las timpanoplastías en 5 tipos ${ }^{1}$.

En Chile, entre los años 1956 y 1959, Maspetiol, Barroilhet, Emhart y Otte $\mathrm{G}$ entre otros, publicaban sus experiencias en la Revista Chilena de Otorrinolaringología $a^{2-5}$.

Durante la década del ochenta los éxitos anatómicos variaban entre $50 \%$ y $72 \%$, donde destacan las publicaciones de Darritchon, Plaza, Caro y Santa María, entre otros ${ }^{6-9}$.

En 1990 se reportó 96\% de éxito anatómico y $77 \%$ funcional, con técnica retroauricular e injerto de periostio de mastoides ${ }^{10}$.

En la última década se han publicado diversos trabajos que muestran un éxito anatómico entre 79\% y $90 \%$ y un éxito auditivo entre $62 \%$ y $92 \%{ }^{11-14}$ (Figura 1 ).

Dentro de la residencia de tercer año de la beca de otorrinolaringología de la Pontificia Universidad Católica de Chile se realiza el comité de oído en el
Figura 1. Comparación éxito anatómico y auditivo de timpanoplastías en la última década.

\begin{tabular}{|c|c|c|c|c|}
\hline AÑo & N & PERIODO & ÉXITO ANATOMICO & $\begin{array}{l}\text { ÉXITO AUDITIVO TOTAL } \\
\text { (MAYOR } 10 \mathrm{~dB} \text { - IDEM) }\end{array}$ \\
\hline $\begin{array}{l}2001 \\
\text { V Vergara Ty cols. } \\
\text { Rev Otorinolaringol. } \\
\text { Cir. Cabeza Cuelio } \\
\text { 2001; 61: 102-106. }\end{array}$ & 113 & $\begin{array}{l}4 \text { AÑOS } \\
(1996- \\
1999)\end{array}$ & $90 \%$ & $85 \%(56 \%-29 \%)$ \\
\hline $\begin{array}{l}2003 \\
\text { MP Vallejos y cols, } \\
\text { Rev. Ootorinolaringol } \\
\text { Cir. Cabeza Cuello } \\
2003 ; 63: 100-105 \text {. }\end{array}$ & $\begin{array}{l}118 \text { (MAY } \\
50 \text { años) }\end{array}$ & $\begin{array}{l}10 \text { AÑOS } \\
\text { (1990- } \\
2000)\end{array}$ & $79 \%$ & $92 \%(36 \%-56 \%)$ \\
\hline 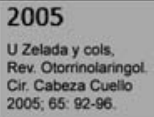 & $\begin{array}{l}233 \\
\text { (NINIOS) }\end{array}$ & $\begin{array}{l}10 \text { AÑOS } \\
\text { (1991- } \\
2000)\end{array}$ & $81 \%$ & $62 \%$ CIERRE GAP \\
\hline $\begin{array}{l}2007 \\
\text { c Toro y cols. } \\
\text { Rev. Otorinolaringol. } \\
\text { Cir. Cabeza Cuellio } \\
\text { 2007; 67. 237-243. }\end{array}$ & 240 & $\begin{array}{l}10 \text { AÑOS } \\
\text { (1995- } \\
2005)\end{array}$ & $89 \%$ & $83 \%(47 \%-36 \%)$ \\
\hline
\end{tabular}


Hospital Clínico UC y el Complejo Asistencial Dr. Sótero del Río (CASR) lo que permite tener diferentes experiencias y en base a esto y el deseo de conocer la experiencia del Complejo Asistencial Dr. Sótero del Río, revisamos los resultados de las timpanoplastías realizadas en un plazo de 5 años.

\section{OBJETIVOS}

Los objetivos de este estudio son identificar y describir las características epidemiológicas de los pacientes sometidos a timpanoplastía atendidos en el CASR, además evaluar la frecuencia de utilización de diferentes técnicas quirúrgicas y sus resultados anatómicos y auditivos.

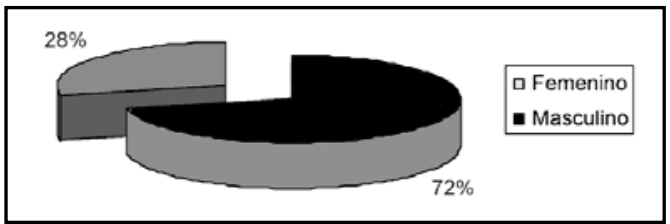

Figura 2. Distribución por sexo.

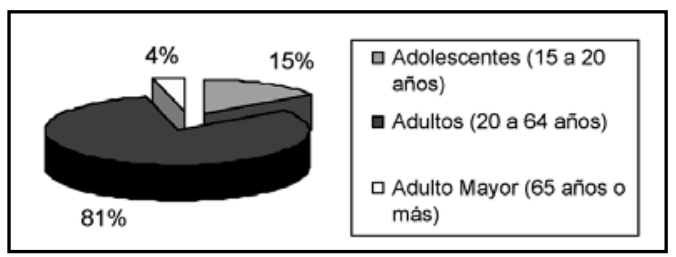

Figura 3. Distribución por rango de edad.

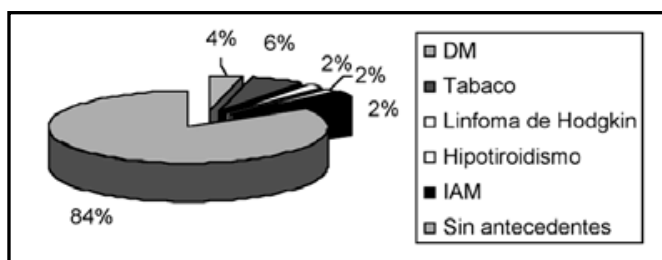

Figura 4. Antecedentes mórbidos.

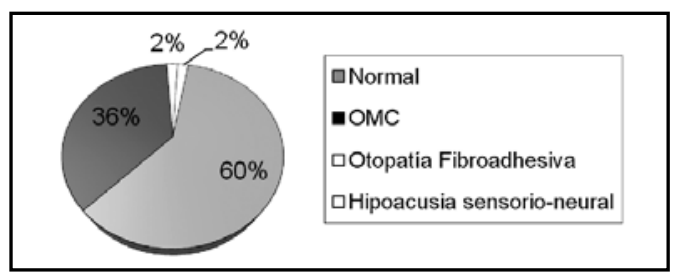

Figura 5. Estado de oído contralateral.

\section{MATERIAL Y MÉTODO}

Se realizó un estudio retrospectivo y descriptivo.

Se revisaron las fichas de los pacientes mayores de 15 años sometidos a timpanoplastía atendidos en el CASR entre enero de 2000 y diciembre de 2004.

Los criterios de inclusión fueron:

- Diagnóstico de OMC no colesteatomatosa

- Audiometría preoperatoria

- Protocolo quirúrgico completo

- Audiometría posoperatoria

- Control, por lo menos de 6 meses, poscirugía

Los criterios de exclusión fueron: 1) cirugías funcionales poscirugía radical de oído y 2) perforación timpánica traumática.

Se registraron edad, sexo, oído operado, estado de oído contralateral, antecedentes mórbidos, promedio de tonos puros (PTP) a los $500,1.000 \mathrm{y}$ $2.000 \mathrm{~Hz}$, tanto de vía ósea como aérea, pre y postimpanoplastía, la técnica quirúrgica utilizada y si el cirujano fue residente 0 staff.

\section{RESULTADOS}

Entre enero de 2000 y diciembre de 2004, se realizaron un total de 101 timpanoplastías de las cuales 53 cumplieron con los criterios de inclusión.

La mayoría de los 48 pacientes no incluidos, no contaba con datos de seguimiento posoperatorio.

El $72 \%$ de los pacientes eran de sexo masculino (Figura 2). En relación a la distribución por rango de edad: $15 \%$ correspondía a adolescentes (entre 15 a 20 años), 81\% correspondía a adultos (entre 20 y 64 años) y sólo $5 \%$ eran adultos mayores (65 años o más) (Figura 3).

La gran mayoría de los pacientes no tenía antecedentes mórbidos, lo que correspondió a $84 \%$ de la muestra total (Figura 4).

Al analizar el estado del oído contralateral, el $60 \%$ de los pacientes tenía un oído normal, el 36\% tenía una otitis media crónica, en tanto otopatía fibroadhesiva e hipoacusia sensorio neural representaban $2 \%$ del total cada una (Figura 5).

El diagnóstico preoperatorio fue otitis media crónica en el $96 \%$ de los casos, el $4 \%$ restante presentaba otopatía fibroadhesiva (Figura 6). 
En el $64 \%$ de los casos el oído operado fue el derecho (Figura 7).

En relación a la vía de abordaje, $74 \%$ fue retroaural y $26 \%$ endoaural (Figura 8 ).

En $58 \%$ de los pacientes el injerto utilizado fue fascia de músculo temporal, en $36 \%$ se utilizó cartílago-pericondrio y en el 6\% restante sólo pericondrio (Figura 9)

La técnica utilizada fue lateral o House en $92 \%$ y medial o Austin en $6 \%$, en un caso se usó técnica mixta en isla (Figura 10).

En relación al tipo de timpanoplastía realizada, ésta fue en $81 \%$ tipo I y en el $19 \%$ restante tipo IIIA (Figura 11).

El resultado anatómico fue en $79 \%$ de los casos tímpano indemne, perforación en $17 \%$, observándose estenosis del conducto auditivo externo (CAE) y lateralización del injerto en $2 \%$ respectivamente (Figura 12).

El éxito anatómico según la vía de abordaje, fue de $79 \%$ cuando fue endoaural y de $77 \%$ cuando la vía utilizada fue retroauricular (Figura 13).

Según el tipo de injerto, el éxito anatómico se observó en $81 \%$ al usar fascia de músculo temporal, $68 \%$ al usar injerto compuesto y en $33 \%$ cuando el injerto usado fue pericondrio (Figura 14).

Al evaluar el éxito anatómico según la colocación del injerto, se evidenció éxito anatómico en 38 de los 49 pacientes operados utilizando técnica House, lo que representa $78 \%$ de éxito. Dos de los tres pacientes operados mediante técnica Austin evidenciaron un buen resultado anatómico poscirugía, lo que equivale al $66 \%$ de los pacientes. El único paciente operado mediante técnica mixta tuvo también un buen resultado anatómico, lo que representa $100 \%$ de éxito en su categoría (Figura 15).

El seguimiento posoperatorio realizado fue de 6 meses en $17 \%$ de los casos, de 6 meses a 2 años en $34 \%$ de los pacientes, entre 2 y 5 años en $26 \%$ de la muestra y mayor a 5 años en $23 \%$, lo que demuestra un muy buen control posoperatorio (Figura 16).

En relación al resultado auditivo, $62 \%$ de los pacientes mejoró más de $10 \mathrm{db}$ su audición y $30 \%$ la mantuvo igual, lo que determina $92 \%$ de los pacientes con buen resultado auditivo. Sólo 8\% empeoró su audición (Figura 17).

En $60 \%$ de las intervenciones el primer cirujano fue un residente de otorrinolaringología, el $40 \%$ restante fue operado por staff (Figura 18).

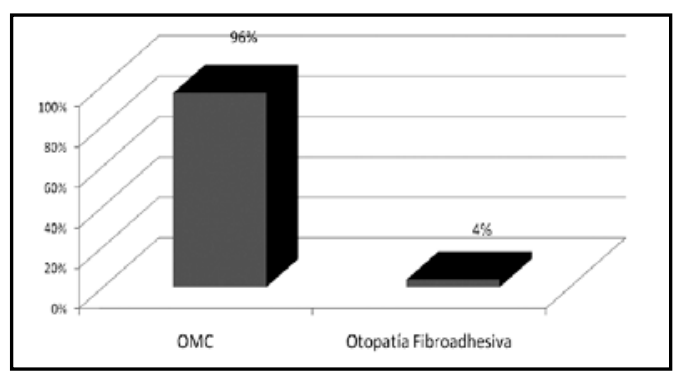

Figura 6. Diagnóstico preoperatorio.

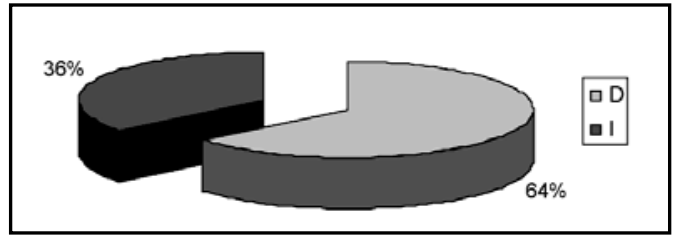

Figura 7. Oído operado.

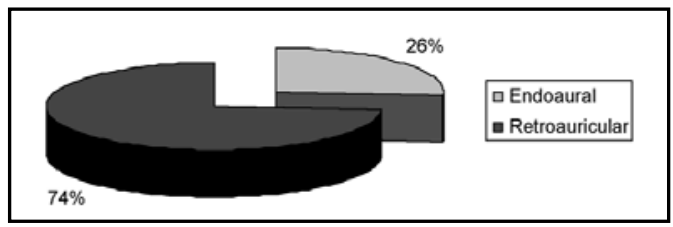

Figura 8. Vía de abordaje.

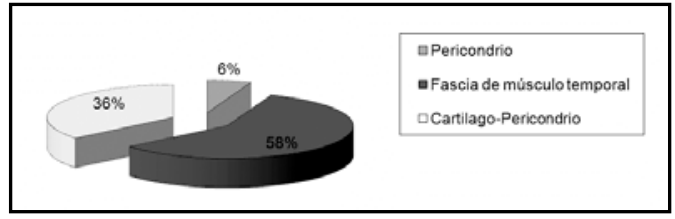

Figura 9. Tipo de injerto.

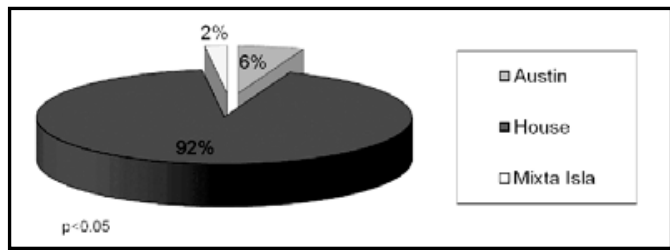

Figura 10. Técnica utilizada: medial o Austin/lateral o House.

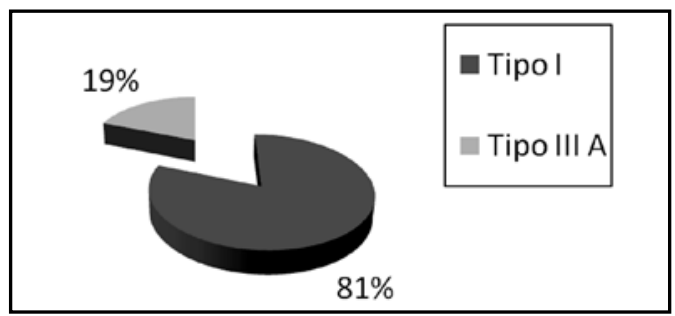

Figura 11. Tipo de timpanoplastía. 


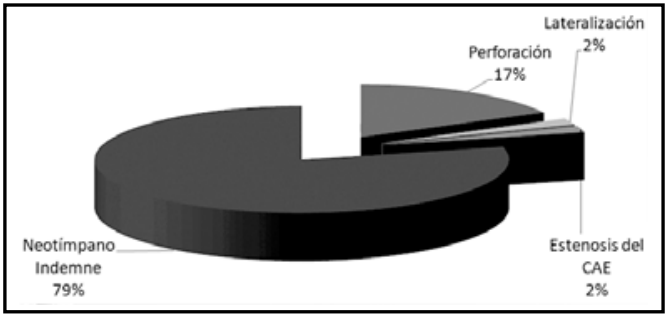

Figura 12. Resultado anatómico.

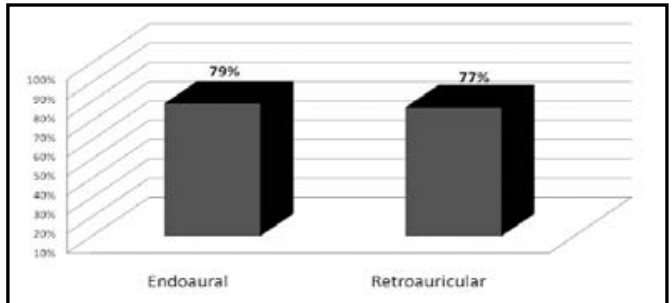

Figura 13. Éxito anatómico según vía de abordaje.

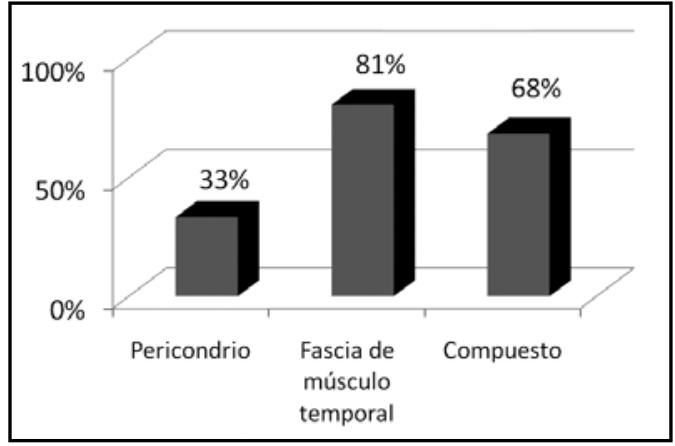

Figura 14. Éxito anatómico según tipo de injerto.

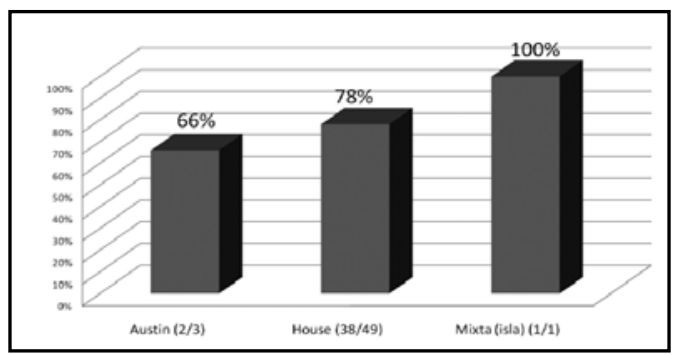

Figura 15. Éxito anatómico según colocación de injerto.

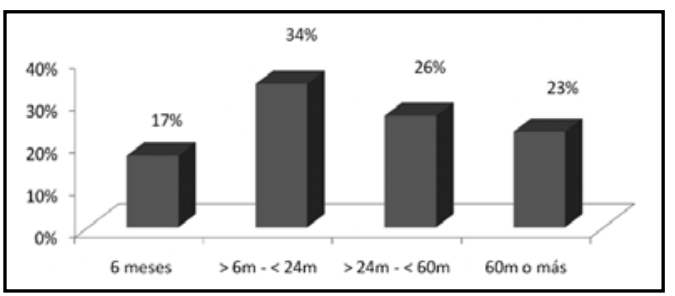

Figura 16. Seguimiento posoperatorio (meses).
En relación a los porcentajes de perforación, las timpanoplastías realizadas por un residente se perforaron en $25 \%$, comparado con $19 \%$ de perforación en las realizadas por un staff (Figura 19).

\section{DISCUSIÓN Y CONCLUSIONES}

Los resultados de reparación anatómica y audiométricos obtenidos de las timpanoplastías realizadas en pacientes mayores de 15 años en nuestro servicio son comparables a los resultados publicados en la literatura nacional e internacional|11,12,14.

El mayor número de intervenciones se realizó en el grupo entre 20 y 64 años de edad (81\%), correspondiendo el $72 \%$ a cirugías realizadas en pacientes de sexo femenino, lo cual ha sido descrito en otros trabajos nacionales ${ }^{11,12,14}$.

La mayoría de los pacientes no presentaban antecedentes mórbidos y en los que éstos existían no fueron estadísticamente significativos.

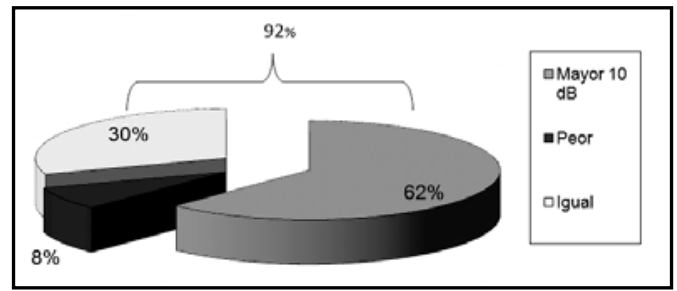

Figura 17. Resultado auditivo.

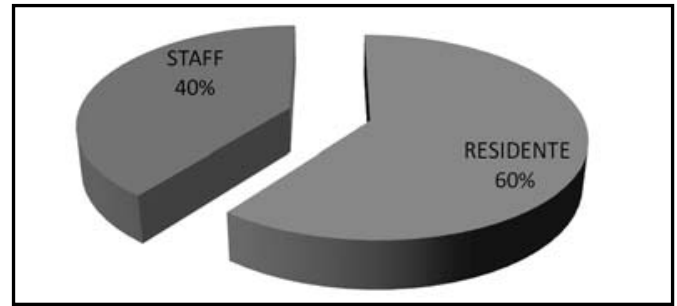

Figura 18. Primer cirujano.

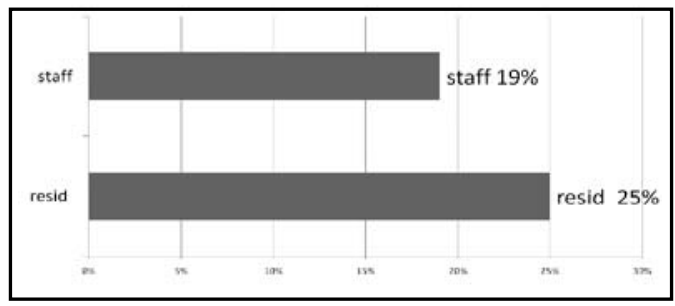

Figura 19. Porcentaje de perforación. 
Se utilizó la vía retroauricular en $74 \%$ y en ellos se utilizó en $58 \%$ injerto de fascia del músculo temporal.

La colocación del injerto se realizó en su mayoría por vía lateral.

Los tipos de timpanoplastía realizada, según la clasificación de Wüllstein, fueron la tipo I y la tipo III.

La reparación de la membrana timpánica se logró en $79 \%$ de los pacientes.

Por otra parte, en el $92 \%$ de los casos se logró mantener o mejorar la audición.

Las complicaciones más frecuentes fueron la perforación (17\%), lateralización (2\%) y estenosis de $\operatorname{CAE}(2 \%)$, siendo su frecuencia similar a 10 descrito en la literatura.

Estos resultados corresponden al rendimiento de la técnica practicada en su mayoría por residentes de $3^{\circ}$ año de ORL y ORL generales con distintos niveles de formación en otocirugía. Esto podría explicar la leve disminución en el éxito anatómico, comparado con series nacionales.

\section{BIBLIOGRAFÍA}

1. Albert Mudry, MD, PhD, Lausanne, Switzerland. History of myringoplasty and tympanoplasty type I. Otolaryngology Head and Neck Surgery 2008; 139: 613-14.

2. Maspetiol R. Indicaciones y técnica de la timpanoplastía (RR). Rev Otorrinolaringol 1956; $16: 17$.

3. Barroilhet J. Nuestra experiencia en microcirugía del oído medio. Rev otorrinolaringol 1958; 27: 38.
4. Emhart 0. Contribución al estudio de las timpanoplastías. Rev Otorrinolaringol 1959; 19: 109.

5. Otte Gabler J, Emhart 0, Cohn M. Timpanoplastías. Otitis media crónica supurada y su tratamiento quirúrgico. Rev Otorrinolaringol 1959; 19: 20.

6. DarRitchon E. Evaluación de Timpanoplastías. Rev Otorrinolaringol 1978; 38: 72-4.

7. Plaza E. Análisis crítico de las timpanoplastías. Rev Otorrinolaringol Cir Cab Cue 1980; 40: 11-6.

8. Caro J y cols. Análisis de 168 timpanoplastías. Rev Otorrinolaringol Cir Cab Cu 1983; 40: 17-23.

9. Santa María A y cols. Resultados de timpanoplastía en niños con tímpano contralateral alterado. Rev Otorrinolaringol 1987; 47: 111-5.

10. Hess J, Rosemblut A. Timpanoplastías. Evaluación de resultados a mediano plazo. Rev Otorrinolaringol Cir Cab Cue 1990; 50: 9-12.

11. V Vergara T, M Arancibia S, J Maass 0 , L Tapia F, M Cumsille. Timpanoplastías: Revisión de 4 años. Rev Otorrinolaringol Cir Cab Cu 2001; 61: 102-6.

12. MP Vallejos, L Dentone. Timpanoplastías en mayores de 50 años. Rev Otorrinolaringol Cir Cab Cu 2003; 63: 100-5.

13. U Zelada, R Arregui, E Palacios. Timpanoplastía en niños: Experiencia de 10 años. Rev Otorrinolaringol Cir Cab Cu 2005; 65: 92-96

14. C Toro, A Naser, C Sanhueza, C Valdés, JP Gormaz, V Molina, A Leslie. Timpanoplastías en adultos en el Hospital Clínico de la Universidad de Chile: Revisión de 10 años. Rev Otorrinolaringol Cir Cab Cu 2007; 67: 237-43. 